\title{
Determinants of COVID-19 disease severity in patients with underlying rheumatic disease
}

\author{
C. Sieiro Santos ${ }^{1}$ - C. Moriano Morales ${ }^{1}$ - E. Díez Álvarez ${ }^{1}$ C. Álvarez Castro ${ }^{1}$ - A. López Robles ${ }^{1} \cdot$ T. Perez Sandoval $^{1}$
}

Received: 4 June 2020 / Revised: 14 July 2020 / Accepted: 17 July 2020 / Published online: 27 July 2020

(C) International League of Associations for Rheumatology (ILAR) 2020

\begin{abstract}
Background Over the month of April, Spain has become the European country with more confirmed cases of COVID-19 infection, after surpassing Italy on April 2nd. The community of Castile and León in Spain is one of the most affected by COVID-19 infection and the province of León has a total of 3711 cases and 425 deaths so far. Rheumatic patients should be given special attention regarding COVID-19 infection due to their immunocompromised state resulting from their underlying immune conditions and use of targeted immune-modulating therapies. Studying epidemiological and clinical characteristics of patients with rheumatic diseases infected with SARS-CoV2 is pivotal to clarify determinants of COVID-19 disease severity in patients with underlying rheumatic disease.

Objectives To describe epidemiological characteristics of patients with rheumatic diseases hospitalized with COVID-19 and determine risk factors associated with mortality in a third level Hospital setting in León, Spain.

Methods We performed a prospective observational study, from 1st March 2020 until the 1st of June including adults with rheumatic diseases hospitalized with COVID-19 and performed a univariate and multivariate logistic regression model to estimate ORs and 95\% CIs of mortality. Age, sex, comorbidities, rheumatic disease diagnosis and treatment, disease activity prior to infection, radiographic and laboratorial results at arrival were analysed.

Results During the study period, 3711 patients with COVID-19 were admitted to our hospital, of whom 38 (10\%) had a rheumatic or musculoskeletal disease. Fifty-three percent were women, with a mean age at hospital admission of 75.3 (IQR 68-83) years. The median length of stay was 11 days. A total of 10 patients died (26\%) during their hospital admission. Patients who died from COVID-19 were older (median age 78.4 IQR 74.5-83.5) than those who survived COVID-19 (median age 75.1 IQR 69.3-75.8) and more likely to have arterial hypertension (9 [90\%] vs 14 [50\%] patients; OR 9 (95\% CI 1.0-80.8), $p 0.049$ ), dyslipidaemia (9 (90\%) vs 12 (43\%); OR 12 (95\% CI 1.33-108), p 0.03), diabetes ((9 (90\%) vs 6 (28\%) patients; OR 33, $p$ $0.002)$, interstitial lung disease (6 (60\%) vs 6 (21\%); OR 5.5 (95\% CI 1.16-26), $p$ 0.03), cardiovascular disease (8 (80\%) vs 11 (39\%); OR 6.18 (95\% IC 1.10-34.7, $p$ 0.04) and a moderate/high index of rheumatic disease activity (7 (25\%) vs 6(60\%); OR 41.4 (4.23-405.23), $p$ 0.04). In univariate analyses, we also found that patients who died from COVID-19 had higher hyperinflammation markers than patients who survived: C-reactive protein (181 (IQR 120-220) vs 107.4 (IQR 30-150; $p$ 0.05); lactate dehydrogenase (641.8 (IQR 465.75-853.5) vs 361 (IQR 250-450), $p$ 0.03); serum ferritin (1026 (IQR 228.31536.3 ) vs 861.3 (IQR 389-1490.5), p 0.04); D-dimer (12,019.8 (IQR 843.5-25,790.5) vs 1544.3 (IQR 619-1622), $p$ 0.04). No differences in sex, radiological abnormalities, rheumatological disease, background therapy or symptoms before admission between deceased patients and survivors were found. In the multivariate analysis, the following risk factors were associated with mortality: rheumatic disease activity $(p=0.003)$, dyslipidaemia $(p=0.01)$, cardiovascular disease $(p=0.02)$ and interstitial lung disease $(p=0.02)$. Age, hypertension and diabetes were significant predictors in univariate but not in multivariate analysis. Rheumatic disease activity was significantly associated with fever $(p=0.05)$, interstitial lung disease $(p=0.03)$, cardiovascular disease $(p=0.03)$ and dyslipidaemia $(p=0.01)$.
\end{abstract}

C. Sieiro Santos

cristysieirosantos@gmail.com

1 Rheumatology Department, Complejo Asistencial Universitario de León, Leon, Spain 
Conclusions Our results suggest that comorbidities, rheumatic disease activity and laboratorial abnormalities such as C-reactive protein (CRP), D-Dimer, lactate dehydrogenase (LDH), serum ferritin elevation significantly associated with mortality whereas previous use of rheumatic medication did not. Inflammation is closely related to severity of COVID-19.

\section{Key Points}

- Most patients recover from COVID-19.

- The use of DMARDs, corticosteroids and biologic agents did not increase the odds of mortality in our study.

- Rheumatic disease activity might be associated with mortality.

Keywords Biologic $\cdot$ COVID-19 $\cdot$ Disease activity $\cdot$ Hospitalized $\cdot$ Hydroxychloroquine $\cdot$ Immunosuppressive $\cdot$ Inflammation $\cdot$ Mortality $\cdot$ Rheumatology $\cdot$ Spain

\section{Introduction}

SARS-CoV-2 infection has spread rapidly across the planet. It is thought to have originated in China's Wuhan province; however, it has spread to more than 140 countries on 6 continents, according to the WHO. Over $80 \%$ of SARS-CoV-2 infection cases have flu-like symptoms; however, $20 \%$ require hospitalization and 5-15\% intensive care [1].

Patients with rheumatic patients deserve special attention since they are considered at-risk for serious infections due to their immunocompromised state resulting from their underlying immune conditions and use of targeted immune-modulating therapies such as biologics and immunomodulatory therapies [2]. Some drugs that modify the activity of the disease, such as hydroxychloroquine, used as treatment of rheumatic diseases have been used for the treatment of COVID-19 [3]. The use of biological therapies such as IL-6 (tocilizumab, sarilumab) and IL-1 (anakinra) inhibitors are also being implemented in patients who developed pathological immune responses such as cytokine storm [4]. It is not clear whether the use of immunosuppressive medication for rheumatic pathology may suppose a risk of developing SARS-CoV-2 infection, if rheumatic patients have a higher mortality rate than general population or what factors may be associated with COVID-19 severity [5].

\section{Methods}

All patients with rheumatic diseases with positive test for SARS-CoV2 admitted to the hospital from the community of León were included in the study. For the purpose of this study, we included patients: (a) aged $>18$ years old, (b) with medical diagnosis of inflammatory rheumatic disease, (b) with SARS-CoV-2 PCR positive diagnostic test or positive antibody serology and (c) patients that required hospitalization. We excluded patients who did not require hospitalization or had a negative test for SARS-CoV2.

Registry data elements included the following co-variables that were considered: (1) sociodemographic baseline characteristics: including sex and age; (2) type of inflammatory rheumatic disease diagnosis: including systemic autoimmune conditions (polymyalgia rheumatica (PMR), vasculitis, systemic sclerosis (SS), Sjogren's syndrome ( $\mathrm{SjS}$ ), systemic lupus erythematosus (SLE) and chronic inflammatory arthritis (rheumatoid arthritis (RA) inflammatory polyarthritis, psoriatic arthritis (PA) and ankylosing spondyloarthritis (AS). (3) Baseline comorbid conditions including hypertension, dyslipidaemia, hyperuricemia, diabetes mellitus, heart disease (including valvulopathies, arrythmias, myocardiopathies, heart failure or pericarditis) and interstitial lung disease. (4) Treatment for inflammatory rheumatic disease: glucocorticoids, conventional synthetic disease-modifying antirheumatic drugs (csDMARDs) including antimalarials (chloroquine/ hydroxychloroquine); sulfasalazine; leflunomide; methotrexate; azathioprine or mycophenolate mofetil, bDMARDs including targeted synthetic or biologic (ts/bDMARDs), including: anti-TNF agents and other Biologics: anti-IL6 (tocilizumab, sarilumab); rituximab; abatacept. (5) Rheumatic disease activity prior to admission. We also compiled information on COVID-19-related illness including diagnosis date, symptoms, laboratory and radiological finding, treatment, the need for ICU care, subsequent complications and death during follow-up.

We performed a univariate and multivariate logistic regression model to estimate ORs and 95\% CIs of mortality. Age, sex, comorbidities, rheumatic disease diagnosis, treatment for rheumatic disease and disease activity prior to infection, duration of hospital stay, symptoms before admission, radiographic abnormalities and laboratorial results at arrival were analysed. 


\section{Radiographic score}

We defined radiographic abnormalities using the following classification system (0-3): 0 for no lung abnormalities, 1 for unilateral interstitial abnormalities, 2 for bilateral interstitial abnormalities and 3 for interstitial and alveolar infiltrates.

\section{Hyperinflammation score}

The presence of hyperinflammation was defined as lymphocyte counts lower than 1000 cells per $\mathrm{mL}$, serum ferritin higher than $500 \mathrm{ng} / \mathrm{mL}$, lactate dehydrogenase higher than $300 \mathrm{U} / \mathrm{L}, \mathrm{C}$-reactive protein higher than 100 and D-dimers higher than $1000 \mathrm{ng} / \mathrm{mL}$.

\section{Rheumatic disease activity}

We revised consultation charts and calculated rheumatic disease activity prior to infection and used the following classification system: 0 for remission, 1 for low/minimal, 2 for moderate, 3 for high/severe.

\section{Medical treatment for COVID-19}

The medical treatment was prescribed according to our hospital's guidelines. Hydroxychloroquine was prescribed at $400 \mathrm{mg}$ bid the first day, followed by $200 \mathrm{mg}$ bid for total of 5 days. Lopinavir/ritonavir 200/50 mg bid was initially indicated in patients with moderate to severe infection. Treatment with systemic steroids and/or biologics (tocilizumab or anakinra) was started in case of worsening of respiratory symptoms or acute respiratory distress syndrome.

\section{Ethics}

The study was conducted according to the principles of the Declaration of Helsinki and approved by our hospital's institutional ethics committee.

\section{Statistical analysis}

Categorical variables were reported as percentages, whereas continuous variables were expressed as median and IQR values. Quantitative variables were compared by use of the non-parametric Mann-Whitney test. Categorical variables were compared by use of contingency tables and $p$ values were calculated with $\chi^{2}$ or Fisher's exact tests, when appropriate.

$p$ values less than 0.05 were considered significant. The effect size for retrospective studies was then evaluated with odds ratios (ORs) with $95 \%$ CIs.

\section{Results}

The demographic and clinical characteristics of the 38 cases in our registry are shown in Table 1 . During the study period, 3711 patients with COVID-19 were admitted to our hospital, of whom $38(10 \%)$ had a rheumatic or musculoskeletal disease. Fifty-three percent were women, with a mean age at hospital admission of 75.3 (IQR 68-83) years. The median length of stay was 11 days. A total of 10 patients died (26\%) during their hospital admission. Most patients had important comorbidities: $60 \%$ patients had hypertension, $55 \%$ had dyslipidaemia, $32 \%$ had diabetes mellitus, $50 \%$ had cardiovascular disease (CD), 32\% had interstitial lung disease (ILD). The most frequent symptoms of COVID-19 were dyspnoea (70\%), cough (66\%), fever (50\%), 26\% reported gastrointestinal symptoms (diarrhoea and vomit), $8 \%$ muscle pain, $8 \%$ odynophagia and dysgeusia. Forty-two percent had a previous diagnosis of rheumatoid arthritis (AR), $24 \%$ of polymyalgia rheumatica (PMR), 13\% of systemic lupus erythematosus (SLE), $8 \%$ of psoriatic arthritis (PA), 5\% of ankylosing spondylitis (AS), $5 \%$ of giant cell arteritis and there was one case of limited systemic sclerosis and Sjogren's disease. At the time of SARS-CoV2 infection, 58\% received oral corticosteroids at a mean dose of $12.65 \mathrm{mg} /$ day, $45 \%$ were taking csDMARDs, $5 \%$ received treatment with bDMARDS and $24 \%$ were taking hydroxychloroquine. Ten patients died. Patients who died from COVID-19 were older (median age 78.4 IQR 74.583.5) than those who survived COVID-19 (median age 75.1 IQR 69.3-75.8) and more likely to have arterial hypertension (9 [90\%] vs 14 [50\%] patients; OR 9 (95\% CI 1.0-80.8), $p$ $0.049)$, dyslipidaemia (9 (90\%) vs 12 (43\%); OR 12 (95\% CI $1.33-108), p 0.03)$, diabetes $((9(90 \%)$ vs $6(28 \%)$ patients; OR 33 (95\% IC 3.46-314.55 $p 0.002$ ), interstitial lung disease (6 (60\%) vs 6 (21\%); OR 5.5 (95\% CI 1.16-26), $p 0.03$ ), cardiovascular disease (8 (80\%) vs 11 (39\%); OR 6.18 (95\% IC 1.10-34.7), $p$ 0.04) and a moderate/high index of rheumatic disease activity (7 (25\%) vs 6(60\%); OR 41.4 (4.23405.23), $p$ 0.04). In univariate analyses, we also found that patients who died from COVID-19 had higher hyperinflammation markers than patients who survived: Creactive protein (181 (IQR 120-220) vs 107.4 (IQR 30-150; $p 0.05$ ); lactate dehydrogenase (641.8 (IQR 465.75-853.5) vs 361 (IQR 250-450), $p$ 0.03); serum ferritin (1026 (IQR 228.3-1536.3) vs 861.3 (IQR 389-1490.5), $p$ 0.04); Ddimer (12,019.8 (IQR 843.5-25,790.5) vs 1544.3 (IQR 619$1622), p 0.04)$. No differences in sex, radiological abnormalities, rheumatological disease or background therapy or symptoms before admission between deceased patients and survivors were found. Glucocorticoids were used for severe respiratory manifestations related to lung involvement in 19 patients $(50 \%)$ and tocilizumab in $3(80 \%)$. To control for possible confounding variables, sequential multivariate regression analyses were performed. In the multivariate analysis, 
Table 1 Demographic and clinical characteristics of SARS-CoV2 with rheumatic disease

\begin{tabular}{|c|c|c|c|c|}
\hline & Survivors $(n=28)$ & Deceased $(n=10)$ & OR $(95 \% \mathrm{CI})$ & $\begin{array}{l}p \\
\text { value }\end{array}$ \\
\hline Age (years) & $75.1(69.3-75.8)$ & $78.4(74.5-83.5)$ & & 0.03 \\
\hline Age $>65$ years & $25(66 \%)$ & $9(90 \%)$ & & \\
\hline \multicolumn{5}{|l|}{ Sex } \\
\hline Male & $39 \%$ & $70 \%$ & $3.6(0.77-17.0)$ & 0.11 \\
\hline Female & $61 \%$ & $30 \%$ & & \\
\hline \multicolumn{5}{|l|}{ Rheumatological diagnosis } \\
\hline RA & $11(39 \%)$ & $5(50 \%)$ & & 0.56 \\
\hline PMR & $6(21 \%)$ & $2(20 \%)$ & & 0.92 \\
\hline SLE & $4(14 \%)$ & $1(10 \%)$ & & 0.73 \\
\hline PA & $3(11 \%)$ & - & & 0.49 \\
\hline AS & $1(4 \%)$ & $1(10 \%)$ & & 0.45 \\
\hline GCA & $2(7 \%)$ & - & & 0.67 \\
\hline Sjogren & $1(4 \%)$ & - & & 0.94 \\
\hline LSS & $1(4 \%)$ & - & & 0.94 \\
\hline \multicolumn{5}{|l|}{ Rheumatological treatment } \\
\hline Glucocorticoids & $18(64 \%)$ & $4(40 \%)$ & & \\
\hline Weekly dosage (mg) & $11.25(5-20) \mathrm{mg}$ & $18.75(8.75-27.5) \mathrm{mg}$ & $\begin{array}{l}0.37 \\
\quad(0.08-1.63)\end{array}$ & 0.19 \\
\hline \multicolumn{5}{|l|}{ Conventional synthetic } \\
\hline DMARDs (csDMARDs) & $12(43 \%)$ & $5(50 \%)$ & $\begin{array}{l}1.33 \\
\quad(0.31-5.67)\end{array}$ & 0.69 \\
\hline Methotrexate & $9(32 \%)$ & $5(50 \%)$ & & 0.32 \\
\hline Hydroxychloroquine & $7(25 \%)$ & - & & 0.19 \\
\hline Leflunomide & $1(4 \%)$ & - & & 0.94 \\
\hline Azathioprine & $1(4 \%)$ & - & & 0.94 \\
\hline Mycophenolate mofetil & $1(4 \%)$ & - & & 0.94 \\
\hline Biological DMARDs & $0(0 \%)$ & $2(20 \%)$ & $\begin{array}{l}16.76 \\
(0.73-384.0- \\
7)\end{array}$ & 0.07 \\
\hline Abatacept & - & $1(10 \%)$ & & \\
\hline Rituximab & - & $1(10 \%)$ & & \\
\hline \multicolumn{5}{|l|}{ Comorbidities } \\
\hline Arterial hypertension & $14(50 \%)$ & $9(90 \%)$ & $9(1-80.8)$ & 0.049 \\
\hline Diabetes & $6(21 \%)$ & $9(90 \%)$ & $\begin{array}{l}33 \\
\quad(3.46-314.5- \\
5)\end{array}$ & 0.002 \\
\hline Hyperuricemia & $5(18 \%)$ & $3(30 \%)$ & $\begin{array}{l}1.97 \\
\quad(0.37-10.39)\end{array}$ & 0.42 \\
\hline Dyslipidaemia & $12(43 \%)$ & $9(90 \%)$ & $\begin{array}{l}12 \\
\quad(1.33-108.0- \\
3)\end{array}$ & 0.03 \\
\hline $\mathrm{CD}$ & $11(39 \%)$ & $8(80 \%)$ & $6.18(1.1-34.7)$ & 0.04 \\
\hline ILD & $6(21 \%)$ & $6(60 \%)$ & $5.5(1.16-26.0)$ & 0.03 \\
\hline \multicolumn{5}{|l|}{ Rheumatic disease activity } \\
\hline Remission & $6(21 \%)$ & $1(10 \%)$ & $\begin{array}{l}2.44 \\
\quad(0.52-11.57)\end{array}$ & 0.26 \\
\hline Low/minimal & $15(54 \%)$ & $3(30 \%)$ & $\begin{array}{l}0.37 \\
\quad(0.07-1.74)\end{array}$ & 0.21 \\
\hline Moderate/high/severe & $7(25 \%)$ & $6(60 \%)$ & $4.5(0.97-20.7)$ & 0.04 \\
\hline Mean hospitalization (days) & $9.9(6-14)$ & $13.2(8-18)$ & - & 0.02 \\
\hline \multicolumn{5}{|l|}{ Symptoms before admission } \\
\hline Dyspnoea & $19(68 \%)$ & $8(80 \%)$ & $4(0.74-21.35)$ & 0.11 \\
\hline
\end{tabular}


Table 1 (continued)

\begin{tabular}{|c|c|c|c|c|}
\hline & Survivors $(n=28)$ & Deceased $(n=10)$ & OR $(95 \% \mathrm{CI})$ & $\begin{array}{l}p \\
\text { value }\end{array}$ \\
\hline Cough & $19(68 \%)$ & $6(60 \%)$ & $1.5(0.36-6.18)$ & 0.57 \\
\hline Fever & $12(43 \%)$ & $7(70 \%)$ & $3.1(0.66-14.59)$ & 0.15 \\
\hline Diarrhoea & $6(21 \%)$ & $1(10 \%)$ & $\begin{array}{l}0.41 \\
\quad(0.04-3.88)\end{array}$ & 0.44 \\
\hline Vomit & $5(18 \%)$ & - & $\begin{array}{l}0.20 \\
\quad(0.01-4.03)\end{array}$ & 0.29 \\
\hline Myalgia & $3(11 \%)$ & - & $\begin{array}{l}0.35 \\
\quad(0.02-7.32)\end{array}$ & 0.49 \\
\hline Odynophagia & $3(11 \%)$ & - & $\begin{array}{l}0.35 \\
\quad(0.02-7.32)\end{array}$ & 0.49 \\
\hline Dysgeusia & $3(11 \%)$ & - & $\begin{array}{l}0.35 \\
\quad(0.02-7.32)\end{array}$ & 0.49 \\
\hline \multicolumn{5}{|l|}{ Laboratorial findings at arrival } \\
\hline $\mathrm{CRP}, \mathrm{mg} / \mathrm{L}$ & 107.4 (IQR 30-150) & 181. 14 (IQR 120-220) & - & 0.05 \\
\hline $\mathrm{LDH}, \mathrm{UI} / \mathrm{L}$ & 361 (IQR 250-450) & 641.8 (IQR 465.75-853.5) & - & 0.03 \\
\hline Ferritin, ng/mL & 861.3 (IQR 389-1490.5) & 1026 (IQR 228.3-1536.3) & - & 0.04 \\
\hline D-dimer, $\mathrm{g} / \mathrm{mL}$ & 1544.3 (IQR 619-1622) & 12,019.8 (IQR 843.5-25,790.5) & - & 0.04 \\
\hline Lymphocytes, $10^{9}$ per L & 966 (IQR 445-965) & 899.8 (IQR 457.5-1375) & - & 0.245 \\
\hline Fibrinogen, mg/dL & 642 (IQR 511-797) & 614 (IQR 511-682) & - & 0.113 \\
\hline Serum transaminases, UI/L & $\begin{array}{l}25 \% \text { patients had moderate-high elevation } \\
(2-3 x \text { the normal level of AST and ALT })\end{array}$ & $\begin{array}{l}30 \% \text { patients had moderate-high elevation } \\
(2-3 x \text { the normal level of AST and ALT) }\end{array}$ & - & - \\
\hline \multicolumn{5}{|l|}{ Radiological findings } \\
\hline $\begin{array}{l}\text { Unilateral interstitial } \\
\text { abnormalities }\end{array}$ & $13(46 \%)$ & $1(10 \%)$ & $\begin{array}{l}0.12 \\
\quad(0.01-1.15)\end{array}$ & 0.067 \\
\hline Bilateral interstitial abnormalities & $6(21 \%)$ & $3(30 \%)$ & $\begin{array}{l}1.57 \\
\quad(0.31-7.98)\end{array}$ & 0.59 \\
\hline $\begin{array}{l}\text { Alveolar/Interstitial/ground-glass } \\
\text { abnormalities }\end{array}$ & $9(32 \%)$ & $6(60 \%)$ & $\begin{array}{l}3.17 \\
\quad(0.71-14.09)\end{array}$ & 0.13 \\
\hline \multicolumn{5}{|l|}{ Complications during hospitalization } \\
\hline ARDS & - & $2(20 \%)$ & $\begin{array}{l}16.76 \\
\quad(0.73-384.0- \\
7)\end{array}$ & 0.078 \\
\hline Pleural effusion & $2(7 \%)$ & $3(30 \%)$ & $\begin{array}{l}5.57 \\
\quad(0.77-40.12)\end{array}$ & 0.088 \\
\hline Acute renal failure & $2(7 \%)$ & $1(10 \%)$ & $\begin{array}{l}1.44 \\
\quad(0.12-17.90)\end{array}$ & 0.77 \\
\hline Rhabdomyolysis & - & $2(20 \%)$ & $\begin{array}{l}16.76 \\
\quad(0.73-384.0- \\
7)\end{array}$ & 0.078 \\
\hline Heart failure & $2(7 \%)$ & $1(10 \%)$ & $\begin{array}{l}1.44 \\
\quad(0.12-17.90)\end{array}$ & 0.088 \\
\hline Heart arrhythmia & $2(7 \%)$ & $1(10 \%)$ & $\begin{array}{l}1.44 \\
\quad(0.12-17.90)\end{array}$ & 0.088 \\
\hline Sepsis & $1(4 \%)$ & $2(20 \%)$ & $\begin{array}{l}6.75 \\
\quad(0.54-84.47)\end{array}$ & 0.139 \\
\hline Hyperinflammation & $2(7 \%)$ & $3(30 \%)$ & $\begin{array}{l}5.57 \\
\quad(0.77-40.12)\end{array}$ & 0.088 \\
\hline None & $21(75 \%)$ & - & - & - \\
\hline \multicolumn{5}{|l|}{ SARS-CoV2 treatment } \\
\hline Hydroxychloroquine & $23(82 \%)$ & $8(80 \%)$ & $\begin{array}{l}0.89 \\
\quad(0.14-5.40)\end{array}$ & 0.88 \\
\hline Chloroquine & $6(21 \%)$ & $1(10 \%)$ & $\begin{array}{l}0.41 \\
\quad(0.04-3.88)\end{array}$ & 0.44 \\
\hline Lopinavir/ritonavir & $25(89 \%)$ & $9(90 \%)$ & $\begin{array}{l}1.08 \\
(0.09-11.76)\end{array}$ & 0.063 \\
\hline Antibiotics & $28(100 \%)$ & $10(100 \%)$ & & 0.63 \\
\hline
\end{tabular}


Table 1 (continued)

Survivors $(n=28)$

Deceased $(n=10)$

$2(20 \%)$

$2(20 \%)$
OR $(95 \% \mathrm{CI}) \quad p$

value

0.36

(0.007-19.7-

8)

37

(1.96-697.3-

9)

6.75

(0.54-84.47)

6.75

(0.54-84.47)
0.02

Data are median (IQR) or $n(\%)$

$S L E$ systemic lupus erythematosus, $P M R$ polymyalgia rheumatica, $A S$ ankylosing spondylitis, $P A$ psoriatic arthritis, $G C A$ giant cell arthritis, $R A$ rheumatoid arthritis, $L S S$ limited systemic sclerosis, $D M A R D s$ disease-modifying anti-rheumatic drugs, $S A R S$-CoV-2 severe acute respiratory syndrome coronavirus, $A D R S$ acute distress respiratory syndrome, $C D$ cardiovascular disease, ILD interstitial lung disease

the following risk factors were associated with mortality: rheumatic disease activity $(p=0.003)$, dyslipidaemia $(p=$ $0.01)$, cardiovascular disease $(p=0.02)$ and interstitial lung disease $(p=0.02)$. Age, hypertension and diabetes were significant predictors in univariate but not in multivariate analysis. Rheumatic disease activity was significantly associated with fever $(p=0.05)$, interstitial lung disease $(p=0.03)$, cardiovascular disease $(p=0.03)$ and dyslipidaemia $(p=0.01)$.

\section{Discussion}

This work describes a collection of COVID-19-hospitalized cases among patients with rheumatic diseases in a third level hospital, in León, Spain. We identified factors associated with mortality in patients with underlying rheumatic diseases, including age, sex, comorbidities, rheumatic diagnosis, treatment, rheumatic disease activity, laboratorial/radiographic abnormalities and complications during admission.

Our data showed that, in a real-world setting, there is a $10 \%$ of patients with underlying rheumatic diseases with COVID19 that required hospital admission, mainly elderly patients, with more comorbidities and systemic autoimmune condition.

In accordance with previous studies of COVID-19 in different populations, we found that patients with comorbidities such as hypertension, dyslipidaemia, diabetes, interstitial lung disease and cardiovascular disease and diabetes had higher odds of mortality $[6,7]$.

The COVID-19 Global Rheumatology Alliance has recently published a cohort of patients with COVID-19 and underlying rheumatic disease, with over 600 cases [8]. Compared to that cohort, patients in our study were more likely to be aged older than 65 years and have a higher rate of comorbidities (hypertension, diabetes, and dyslipidaemia) and a more severe disease course. In several studies, the importance of diabetes as comorbidity in COVID-19 has already been seen. It was stated that diabetes is an independent risk factors for morbidity and mortality in COVID-19 [9].

We did not find a significant association between mortality and antimalarial use before hospitalization. Thirty-five percent of the patients were negative for reverse transcriptasepolymerase chain reaction of nasopharyngeal and sputum swabs after receiving treatment; however, almost all of these patients had mild disease and would likely have a favourable clinical outcome and viral clearance regardless of treatment. There has been speculation about the use of HCQ in the treatment of COVID-19 and many randomized studies have been conducted, claiming nasopharyngeal virus clearance in cases treated with antimalarial agents; however, the evidence supporting the use of HCQ is not compelling [10]. Recently, an observational study found no significant benefit with either hydroxychloroquine alone or combined with azithromycin on clinical outcomes including mortality [11].

We also did not detect differences in background therapies when comparing deceased patients with survivors, such as csDMARDS, corticosteroid and biologic agents; however, we found differences in hyperinflammation markers between the two groups and found that patients who died from COVID-19 are more likely to have moderate/high rheumatic disease activity prior to infection.

Several studies have theorized that SARS-CoV2 infection can induce cytokine release, leading to an increase in IL-6, IL10 , and TNF. In cytokine release syndrome, common laboratory abnormalities in hospitalized patients involve elevated liver enzymes, serum ferritin value, C-reactive protein, D-dimer, coagulation times (PT/PTT) and lactate dehydrogenase (LDH) [4]. In fact, we observed that patients with severe prognosis often associate a rate of hyperinflammation or need for respiratory support, which required the use of glucocorticoids or tocilizumab. Fadel et al. have recently pointed out the use of 
corticosteroids in hospitalized patients with moderate/severe COVID-19; however, several other studies found that corticosteroid use may delay the clearance of COVID-19 and leading to virus replication [12]. In our hospital, the use of glucocorticoids or tocilizumab, or both, was based on the severity of respiratory symptoms, in accordance with our hospital's protocol.

The number of patients taking biologic drugs was very small in our study which may be insufficient to demonstrate other underlying effects. There has been talk about the effect of biologic treatment in COVID-19 infection. Several studies have highlighted the use of biologic agents, as evidenced by those with more severe disease having higher levels of cytokines, including IL-6 and TNF [13]. Treatment with anti-IL6 receptor monoclonal antibody, tocilizumab has been shown effective in treating cytokine release. IL-6 levels can be easily measured in blood in hospitalized COVID-19 patients. A recent study showed that elevated IL-6 concentration was associated with detectable serum SARS-CoV2 RNA in COVID19 patients and reflects the severity of the disease. The same study also associates parameters of multiple organ dysfunction, like troponin T levels with higher IL-6 levels [14].

These data reinforce the idea that the prognosis of COVID19 is more likely to be related to the presence of other risk factors rather than the rheumatic and musculoskeletal disease itself or the background therapy; and that hyperinflammation is associated with the severity of COVID-19. The results of our study should be interpreted considering limitations. First, we have included a small sample size with underlying rheumatic diseases who needed hospitalization; second, we should consider the observational nature of the study and that our patients were treated at a single centre.

Overall, the prognosis of patients with rheumatic diseases hospitalized with COVID-19 is good; however, our findings should be interpreted with caution.

Studies including inflammatory rheumatic disease patients with COVID-19 are still limited; however, we hope these data can shed some light as to know which of the factors may be associated with an increased mortality.

\section{Conclusion}

Our results suggest that comorbidities, rheumatic disease activity and laboratorial abnormalities such as $\mathrm{C}$-reactive protein (CRP), D-Dimer, lactate dehydrogenase (LDH), ferritin serum elevation significantly associated with mortality whereas previous use of hydroxychloroquine, corticosteroid, dcDMARDS and biologic therapy did not. Laboratory abnormalities like elevated LDH, CRP, serum ferritin, D-dimer were found more frequently in critically ill patients over the course of hospitalization, suggesting inflammatory cytokine storm is closely related to severity of COVID-19.
Acknowledgments The authors acknowledge the assistance of study participant, radiographers, study nurses and laboratory staff who participated in the study. The study was conducted without any financial support.

\section{Compliance with ethical standards}

Disclosures None.

\section{References}

1. Pan F, Ye T, Sun P, Gui S, Liang B, Li L et al (2020) Time Course of Lung Changes On Chest CT During Recovery From 2019 Novel coronavirus (COVID-19) pneumonia. Radiology.:200370

2. Furst DE (2010) The risk of infections with biologic therapies for rheumatoid arthritis. Semin Arthritis Rheum 39:327-346

3. Kim AHJ, Sparks JA, Liew JW et al (2020) A rush to judgment? Rapid reporting and dissemination of results and its consequences regarding the use of hydroxychloroquine for COVID-19. Ann Intern Med published online March 30

4. Mehta P, McAuley DF, Brown M, Sanchez E, Tattersall RS, Manson JJ, HLH Across Speciality Collaboration, UK (2020) COVID-19: consider cytokine storm syndromes and immunosuppression. Lancet 395:1033-1034

5. Favalli EG, Ingegnoli F, De Lucia O, Cincinelli G, Cimaz R, Caporali R (2020) COVID-19 infection and rheumatoid arthritis: faraway, so close! Autoimmun Rev

6. Goyal P, Choi JJ, Pinheiro LC et al (2020) Clinical characteristics of Covid-19 in New York City. N Engl J Med Overseas Ed. https:// doi.org/10.1056/NEJMc2010419

7. Richardson S, Hirsch JS, Narasimhan M et al (2020) Presenting characteristics, comorbidities, and outcomes among 5700 patients hospitalized with COVID-19 in the New York City area. JAMA. https://doi.org/10.1001/jama.2020.6775

8. Gianfrancesco M, Hyrich KL, Al-Adely S et al (2020; published online May 29) Characteristics associated with hospitalisation for COVID-19 in people with rheumatic disease: data from the COVID-19 Global Rheumatology Alliance physician-reported registry. Ann Rheum Dis. https://doi.org/10.1136/annrheumdis-2020217871

9. Yang JK, Feng Y, Yuan MY, Yuan SY, Fu HJ, Wu BY, Sun GZ, Yang GR, Zhang XL, Wang L, Xu X, Xu XP, Chan JCN (2006) Plasma glucose levels and diabetes are independent predictors for mortality and morbidity in patients with SARS. Diabet Med 23(6): 623-628

10. Graef ER, Liew JW, Putman MS, Simard JF, Sirotich E, Berenbaum F, Duarte-García A, Grainger R, Harrison C, Konig MF, Korsten P, Proulx L, Richards DP, Robinson PC, Sattui SE, Ugarte-Gil MF, Young KJ, Kim AHJ, Sparks JA (2020) Festina lente: hydroxychloroquine, covid-19 and the role of the rheumatologist. Ann Rheum Dis:annrheumdis-2020-217480. https://doi.org/ 10.1136/annrheumdis-2020-217480

11. Mahevas M, Tran VT, Roumier M et al (2020) No evidence of clinical efficacy of hydroxychloroquine in patients hospitalized for COVID-19 infection with oxygen requirement: results of a study using routinely collected data to emulate a target trial. medRxiv

12. Raef Fadel DO, Morrison AR, Pharm D, Amit Vahia MD, Smith ZR, Pharm D, Zohra Chaudhry MD, Pallavi Bhargava MD, Joseph Miller MD, Kenney RM, Pharm D, George Alangaden MD, Mayur S, Ramesh MD, Ford HCOVID-19 Management Task Force, Early Short Course Corticosteroids in Hospitalized Patients with COVID19. Clin Infect Dis:ciaa601. https://doi.org/10.1093/cid/ciaa601 
13. Huang C, Wang Y, Li X et al (2020) Clinical features of patients infected with 2019 novel coronavirus in Wuhan, China. Lancet 395:497-506. https://doi.org/10.1016/S0140-6736(20)30185 pmid:http://www.ncbi.nlm.nih.gov/pubmed/31986264

14. Chen X, Zhao B, Qu Y, Chen Y, Xiong J (2020) Detectable serum SARS-CoV-2 viral load (RNAaemia) is closely correlated with drastically elevated interleukin 6 (IL-6) level in critically ill
COVID-19 patients. Oxford University Press for the Infectious Diseases Society of America

Publisher's note Springer Nature remains neutral with regard to jurisdictional claims in published maps and institutional affiliations. 